Artículo original

\title{
Evaluación del estado trófico de El Salitre, último humedal urbano de referencia en Bogotá
}

\author{
Paulo César Pulido-López ${ }^{1, *}$, Gabriel Antonio Pinilla-Agudelo ${ }^{2}$ \\ ${ }^{1}$ Universidad Jorge Tadeo Lozano, Bogotá, Colombia \\ ${ }^{2}$ Universidad Nacional de Colombia, Bogotá, Colombia
}

\begin{abstract}
Resumen
El Distrito Capital de Bogotá cuenta con 15 humedales reconocidos como parte del sistema de áreas protegidas de la ciudad, la mayoría de los cuales se encuentran seriamente afectados debido a la presión ejercida por el acelerado crecimiento urbano. A diferencia de los otros sistemas del mismo tipo, el humedal El Salitre, recientemente declarado como Parque Ecológico Distrital de Humedal, no se ha visto afectado por vertimientos de aguas residuales. En este estudio se determinó el estado trófico de dicho humedal a partir de la concentración de nutrientes y clorofila a fitoplanctónica, el cálculo de los índices del estado trófico y la composición taxonómica del fitoplancton. El nutriente limitante fue el nitrógeno. Las bajas concentraciones de compuestos nitrogenados, los índices fitoplanctónicos y la dominancia de desmidias (Conjugatophyceae), indicaron que el humedal El Salitre presentó condiciones de oligotrofia a mesotrofia en los dos periodos climáticos durante los cuales se estudió. Los resultados sugieren que este humedal podría servir como sistema de referencia para los demás humedales de Bogotá. (C) 2017. Acad. Colomb. Cienc. Ex. Fis. Nat.
\end{abstract}

Palabras clave: Nutrientes; Clorofila a; Fitoplancton; Sistema palustre; Índices de estado trófico; Humedal conservado.

Evaluation of the trophic state of EI Salitre, the last urban reference wetland of Bogotá

\begin{abstract}
The Capital District of Bogotá has 15 wetlands recognized within the system of protected areas of the city, most of which are seriously affected due to the pressure exerted by the rapid urban growth. Unlike other systems of the same type, El Salitre wetland, recently declared as a Wetland District Ecological Park, has not been affected by wastewater discharges. In this study, we determined its trophic state based on the concentrations of nutrients and phytoplanktonic chlorophyll a, trophic state indices calculation and the taxonomic composition of phytoplankton. Nitrogen was the limiting nutrient. Low concentrations of nitrogen compounds, phytoplankton indices, and dominance of desmids (Conjugatophyceae) indicated that El Salitre wetland presented an oligotrophic to mesotrophic condition in the two climatic periods during which it was studied. These results suggest that it could serve as reference system for other wetlands in Bogotá. (C) 2017. Acad. Colomb. Cienc. Ex. Fis. Nat.
\end{abstract}

Key words: Nutrients; Chlorophyll a; Phytoplankton; Palustrine system; Trophic state index; Preserved wetland.

\section{Introducción}

El humedal El Salitre hace parte de la estructura ecológica principal de la ciudad de Bogotá, pero aún no se tiene información sobre sus características limnológicas, ni cuenta con un Plan de Manejo Ambiental aprobado. Este ecosistema es distinto a la mayoría de humedales de la ciudad, debido a que no presenta vertimientos de aguas residuales y solo es alimentado por aguas lluvias y de escorrentía (EAAB, 2011). En consecuencia, El Salitre tiene condiciones particulares que podrían ser similares a las de los ecosistemas palustres presentes en la Sabana de Bogotá antes del impacto generado por el rápido desarrollo de la ciudad. El extenso sistema de humedales que existió en el pasado reciente (50 mil hectáreas a principios del siglo $\mathrm{XX}$ ) se ha reducido drásticamente y en la actualidad solo permanecen entre 800 (Alcaldía Mayor de Bogotá, 2000) y 1500 hectáreas (Moreno, García \& Villalba, 2005), es decir, que solo se conserva un 2 a $3 \%$ de estos ecosistemas estratégicos. Es por tanto de suma importancia contar con un sistema que sirva como ambiente de referencia en futuros planes de seguimiento y restauración de humedales de la ciudad de Bogotá. En este sentido, un ecosistema de referencia sirve como modelo para restaurar otros ecosistemas, lo cual implica que el sitio de referencia tiene procesos ecológicos mejor conservados,

\footnotetext{
*Correspondencia:

Paulo Pulido-López, pauloc.pulidol@utadeo.edu.co

Recibido: 19 de septiembre de 2016

Aceptado: 20 de febrero de 2017
} 
mayor funcionalidad, estructura más compleja y mayor diversidad que el sistema a restaurar (SER, 2004). Desde una perspectiva urbana, el sistema de referencia puede ser una porción menos perturbada de una matriz más alterada.

Un aspecto limnológico importante en el estudio de los ecosistemas acuáticos es el estado trófico, el cual se relaciona con las características fisicoquímicas del agua, principalmente con la concentración de nutrientes (nitrógeno y fósforo) y con la abundancia y composición de las comunidades planctónicas. De éstas, el fitoplancton permite conocer las condiciones del momento, pues responde rápidamente a diversos cambios en las características abióticas de la columna de agua. Esto se debe al pequeño tamaño de las microalgas y a sus ciclos de vida cortos, que las hacen altamente susceptibles a las variaciones físicas y químicas del agua (Ramírez, 2000; Vásquez, Ariza \& Pinilla, 2006).

Se han realizado estudios sobre el estado trófico de varios humedales de la Sabana de Bogotá (Vásquez, et al., 2006; Malagón, 2008; Pinilla, 2010; López, 2012; Rodríguez, 2012; Beltran \& Rangel, 2013; Rivera-Usme, Pinilla-Agudelo \& Rangel-Ch, 2013), pero en ninguno se incluye el humedal El Salitre. En el presente documento, se describe el estado trófico del humedal El Salitre en dos periodos hidroclimáticos contrastantes, con base en cuatro criterios específicos: la concentración de nutrientes (nitrógeno y fósforo), la concentración de clorofila $a$ fitoplanctónica, la composición del fitoplancton y el cálculo de índices propuestos para ecosistemas lénticos. La hipótesis propuesta, dadas las características de limitada intervención antrópica que presenta este ecosistema, fue que sus aguas tienden hacia un menor contenido de nutrientes, condición que se reflejará en una comunidad fitoplanctónica propia de aguas oligo a mesotróficas.

\section{Materiales y Métodos}

Área de estudio. El humedal El Salitre está ubicado en la localidad de Barrios Unidos (Bogotá D.C., Colombia), dentro del Parque Recreo deportivo El Salitre (440'6”N 74'5'17'W). Es un humedal artificial (DAMA, 2002) que se encuentra a $2600 \mathrm{msnm}$, tiene una extensión de 6,4 hectáreas y su zona inundable es de 13208,85 $\mathrm{m}^{2}$. La profundidad media es menor a $1,5 \mathrm{~m}$. La temperatura promedio anual es de $14,2^{\circ} \mathrm{C}$, con fluctuaciones entre $9 \mathrm{y}$ $22{ }^{\circ} \mathrm{C}$ y la precipitación total es de 900 a1000 mm al año (Alcaldía Local de Barrios Unidos, 2014). El régimen de lluvias, común para toda la Sabana de Bogotá, es bimodal, con dos periodos de máxima precipitación (marzo-mayo y octubre-noviembre) y dos periodos secos (enero-febrero y julio-agosto) (López, 2012).

Los sitios de colección se ubicaron y georreferenciaron en un pre muestreo realizado en noviembre de 2013. Las muestras se tomaron en tres puntos del humedal en febrero (baja precipitación) y mayo (alta precipitación) de 2014. En el costado norte del humedal se ubicó el punto uno $\left(4^{\circ} 40^{\prime} 5,8^{\prime} \mathrm{N} 74^{\circ} 5^{\prime} 7,9^{\prime} \mathrm{W}\right)$. Este sector se encontró en un avanzado proceso de terrificación, con poca profundidad, abundante pasto kikuyo (Pennisetum clandestinum) y gran cantidad de macrófitas sumergidas y flotantes, principalmente Utricularia spp. y Marsilea spp., respectivamente. En el sector medio del humedal se ubicó el punto dos $\left(4^{\circ} 40^{\prime} 4,2^{\prime}\right.$ N $\left.74^{\circ} 5^{\prime} 17,3^{\prime \prime} \mathrm{W}\right)$, el cual tuvo predominio de aguas libres, presencia de algunas macrófitas sumergidas (principalmente Utricularia spp.) y mayor profundidad. En el costado sur del humedal se ubicó el punto tres $\left(4^{\circ} 40^{\prime} 00,4^{\prime}{ }^{\prime} \mathrm{N}\right.$ $74^{\circ} 5^{\prime} 18,0^{\prime} \mathrm{W}$ ), en el que también prevalecieron las aguas libres con macrófitas sumergidas (Utricularia spp.) y una profundidad similar a la del punto dos; el litoral de este sitio estuvo rodeado por juncos (Juncus effusus).

Parámetros fisicoquímicos. La temperatura, el pH, la conductividad eléctrica, el oxígeno disuelto, el porcentaje de saturación de oxígeno y la profundidad del sistema se midieron in situ con equipos portátiles (HACH) siguiendo los protocolos indicados en APHA, AWWA \& WPCF (2012). La transparencia del agua se estableció con un disco Secchi. En cada punto se tomaron además dos muestras de agua con una botella Alpha horizontal de $2 \mathrm{~L}$ a $15 \mathrm{~cm}$ de profundidad, las cuales se preservaron y se refrigeraron a $4^{\circ} \mathrm{C}$ hasta su traslado a un laboratorio acreditado por el IDEAM, en donde se analizaron los nutrientes (nitritos, nitratos, amonio, fosfatos) empleando métodos estándar (APHA, et al., 2012).

Concentración de clorofila a fitoplanctónica. Para este análisis cada muestra se tomó por triplicado con una botella Alpha horizontal de $2 \mathrm{~L}$ a $15 \mathrm{~cm}$ de profundidad y se guardaron en frascos de vidrio color ámbar de $1 \mathrm{~L}$, refrigerándolas a $4{ }^{\circ} \mathrm{C}$ en oscuridad hasta su traslado al laboratorio. La metodología y los cálculos para estimar la concentración de clorofila $a$ del fitoplancton siguieron los procedimientos espectrofotométricos indicados por la Confederación Hidrográfica del Ebro (2005).

Estudio del fitoplancton. Para recolectar las muestras de fitoplancton se siguieron las metodologías propuestas por Wetzel \& Likens (2000), Ramírez (2000) y Roldán \& Ramírez (2008). Las muestras para el análisis cualitativo se obtuvieron por filtración de $30 \mathrm{~L}$ de agua de los primeros 30 $\mathrm{cm}$ a través de una red de fitoplancton de $20 \mu \mathrm{m}$ de criba. Se concentraron $250 \mathrm{~mL}$ de cada muestra, los cuales se fijaron con un volumen igual de solución Transeau (formol $40 \%$, etanol $90 \%$, agua destilada en proporciones 1:3:6 en volumen) en frascos de vidrio de $500 \mathrm{~mL}$. Para este análisis, cada muestra se tomó por duplicado. La observación del fitoplancton se realizó en un microscopio óptico marca Nikon Eclipse 50i. Para la identificación se emplearon claves taxonómicas especializadas como las de Prescott, Croasdale \& Vinyard (1972, 1975, 1977), Prescott, Croasdale, Vinyard \& Bicudo (1981), Prescott, Bicudo \& Vinyard (1982), Parra, González, Dellarossa, Rivera \& Orellana (1982), Tell \& Conforti (1986), Wehr, Sheath \& Kociolek (2003), Bicudo \& Meneses (2006) y Bellinger \& Sigee (2010), entre otras. El sistema de clasificación se basó en Guiry \& Guiry (2015). 
Las muestras para el análisis cuantitativo se tomaron por triplicado con una botella Alpha horizontal de $2 \mathrm{~L}$ a 15 $\mathrm{cm}$ de profundidad. Previa homogenización, se guardaron $400 \mathrm{~mL}$ de muestra en frascos de vidrio de color ámbar y se fijaron con solución de Lugol $(0,5 \mathrm{~mL}$ por cada $100 \mathrm{~mL}$ de muestra). La cuantificación se realizó con el método de sedimentación y observación al microscopio invertido (Karlson, Cusack \& Bresnan, 2010), utilizando cámaras tipo Utermöhl de $10 \mathrm{~mL}$. El periodo de sedimentación fue de 24 h y la observación se hizo en un microscopio invertido marca Advanced Optical en $400 \mathrm{X}$, realizando un conteo parcial mediante el método de campos aleatorios, hasta llegar a un mínimo de 400 individuos de la especie más frecuente, lo cual permite tener un límite de confiabilidad de 0,95 (Wetzel \& Likens, 2000). Al llegar a 400 individuos se realizó el barrido de toda la placa para registrar las morfoespecies que no habían sido observadas. Para esta investigación, se consideró cada individuo algal como una unidad (unicelular, colonial o filamentosa). Los valores se reportan en individuos por $\mathrm{mL}$, para lo cual se empleó la fórmula indicada en APHA, et al. (2012).

Análisis de datos. Se realizó un análisis estadístico descriptivo de cada una de las variables físicas, químicas y biológicas a partir de los promedios de las réplicas obtenidas en campo y observadas en laboratorio. La determinación del estado trófico se hizo con el promedio de los datos de los tres puntos de muestreo, para cada época climática. Se calcularon los índices de Nygaard para el fitoplancton (Ramírez, 2000; Bellinger \& Sigee, 2010), el índice trófico plantónico de Barbe (ITP) (De la Fuente, 2004) y el índice de estado trófico ponderado de Carlson (IET) (Ramírez, 2000). Los cómputos se realizaron en la hoja de cálculo Excel de Microsoft.

\section{Resultados}

Concentración de nutrientes y de clorofila a fitoplanctónica. En la tabla 1 se muestran las concentraciones de los compuestos nitrogenados, los ortofosfatos y la clorofila $a$, y se indica su relación con el estado trófico que presentó el humedal El Salitre durante cada periodo climático, en cada punto de muestreo. Se utilizaron como valores de referencia los indicados por la OCDE (1982) para nitrógeno amoniacal $\left(\mathrm{NH}_{4}^{+}\right)$, nitritos $\left(\mathrm{NO}_{2}^{-}\right)$y nitratos $\left(\mathrm{NO}_{3}^{-}\right)$, por Dabrowski (2014) para ortofosfatos $\left(\mathrm{PO}_{4}{ }^{3-}\right)$, y por Esteves (1998) para clorofila $a(\mathrm{Chl} a)$. Se debe tener en cuenta que estos datos de referencia están dados para ecosistemas lénticos de zonas templadas.

Como se observa, las concentraciones de compuestos nitrogenados indican una condición de oligotrofia a mesotrofia en ambos periodos climáticos, mientras que las concentraciones de clorofila $a$ y de ortofosfatos señalan ambientes eutróficos en los tres puntos de muestreo, en las dos épocas climáticas. Según las cantidades de nitrógeno amoniacal, el estado oligotrófico de la época seca pasó a mesotrófico en el periodo de lluvias, mientras que, con base en los nitritos y los nitratos, el humedal mantuvo su condición oligotrófica en los dos periodos climáticos.

Índices de estado trófico. La tabla 2 presenta el estado trófico que tuvo el humedal El Salitre en los dos periodos climáticos, con base en los índices de Nygaard, de Barbe y de Carlson. Para el cálculo de éste último índice se emplearon los valores de transparencia Secchi $(0,38 \pm 0,06$ y $0,49 \pm 0,09)$ obtenidos en las épocas seca y de lluvias, respectivamente. El índice de diatomeas no se pudo calcular, debido a que no se registraron especies de bacilariofíceas centrales. Los índices cianofíceo, euglenofíceo y compuesto de Nygaard

Tabla 1. Estado trófico del humedal El Salitre en los tres puntos de muestreo según la concentración de nutrientes y de clorofila $a$.

\begin{tabular}{|c|c|c|c|c|c|c|c|c|c|c|}
\hline \multirow[t]{2}{*}{ Sitio } & \multicolumn{2}{|c|}{$\begin{array}{c}\text { Estado Trófico } \\
\text { basado en el amonio }\end{array}$} & \multicolumn{2}{|c|}{$\begin{array}{c}\text { Estado Trófico } \\
\text { basado en el nitrito }\end{array}$} & \multicolumn{2}{|c|}{$\begin{array}{c}\text { Estado Trófico } \\
\text { basado en el nitrato }\end{array}$} & \multicolumn{2}{|c|}{$\begin{array}{c}\text { Estado Trófico } \\
\text { basado en el fosfato }\end{array}$} & \multicolumn{2}{|c|}{$\begin{array}{c}\text { Estado Trófico basado } \\
\text { en la clorofila }\end{array}$} \\
\hline & {$\left[\mathrm{NH}_{4}^{+}\right]$} & Categoría & {$\left[\mathrm{NO}_{2}^{-}\right]$} & Categoría & {$\left[\mathrm{NO}_{3}^{-}\right]$} & Categoría & {$\left[\mathrm{PO}_{4}{ }^{3-}\right]$} & Categoría & [Chl a] & Categoría \\
\hline S_1 & 0,025 & $\mathrm{O}$ & 0,024 & $\mathrm{O}$ & 0,05 & $\mathrm{O}$ & 0,045 & E & 54,32 & E \\
\hline S_2 & 0,025 & $\mathrm{O}$ & 0,014 & $\mathrm{O}$ & 0,05 & $\mathrm{O}$ & 0,045 & $\mathrm{E}$ & 29,98 & E \\
\hline S_3 & 0,025 & $\mathrm{O}$ & 0,011 & $\mathrm{O}$ & 0,05 & $\mathrm{O}$ & 0,045 & $\mathrm{E}$ & 31,61 & $\mathrm{E}$ \\
\hline $\begin{array}{l}\text { Promedio } \\
\text { S }\end{array}$ & $\mathbf{0 , 0 2 5} \pm 0 *$ & $\mathbf{O}$ & $\mathbf{0 , 0 1 6 3} \pm 0,006^{*}$ & $\mathbf{O}$ & $\mathbf{0 , 0 5} \pm 0 *$ & $\mathbf{O}$ & $\mathbf{0 , 0 4 5} \pm 0 *$ & $\mathbf{E}$ & $\mathbf{3 8 , 6 4} \pm 13,6^{*}$ & $\mathbf{E}$ \\
\hline L_1 & 0,46 & M & 0,0035 & $\mathrm{O}$ & 0,05 & $\mathrm{O}$ & 0,13 & $\mathrm{E}$ & 65,28 & $\mathrm{E}$ \\
\hline L_2 & 0,45 & M & 0,0035 & $\mathrm{O}$ & 0,05 & $\mathrm{O}$ & 0,19 & $\mathrm{E}$ & 19,86 & E \\
\hline L_3 & 0,45 & M & 0,0035 & $\mathrm{O}$ & 0,05 & $\mathrm{O}$ & 0,26 & $\mathrm{E}$ & 50,51 & $\mathrm{E}$ \\
\hline $\begin{array}{c}\text { Promedio } \\
\text { L }\end{array}$ & $0,453 \pm 0,005^{*}$ & $\mathbf{M}$ & $\mathbf{0 , 0 0 3 5} \pm 0 *$ & $\mathbf{O}$ & $\mathbf{0 , 0 5} \pm 0 *$ & $\mathbf{O}$ & $\mathbf{0 , 1 9 3} \pm 0,065^{*}$ & $\mathbf{E}$ & $\mathbf{4 5 , 2 1} \pm 23,1^{*}$ & $\mathbf{E}$ \\
\hline
\end{tabular}

$\mathrm{S}=$ época seca, $\mathrm{L}=$ época lluviosa, 1, 2, 3= puntos de muestreo

$\mathrm{O}=$ oligotrófico, $\mathrm{M}=$ mesotrófico, $\mathrm{E}=$ eutrófico

La clasificación se basa en los valores de referencia de OCDE (1982) para nitrógeno amoniacal $\left(\mathrm{NH}_{4}^{+}\right)$, nitritos $\left(\mathrm{NO}_{2}^{-}\right)$y nitratos $\left(\mathrm{NO}_{3}^{-}\right)$, de $\mathbf{D a b r o w s k i}$ (2014) para ortofosfatos $\left(\mathrm{PO}_{4}^{3-}\right)$ y de Esteves (1998) para clorofila $a(\mathrm{Chl} a)$. Las concentraciones de los nutrientes se registran en mg $\mathrm{L}^{-1} \mathrm{y}$ la de clorofila $a$ en $\mathrm{mg} \mathrm{m}^{-3}$

*Desviación estándar de cada variable en cada época de muestreo 
tienen en cuenta la relación entre los taxones de microalgas presentes en el fitoplancton. Según los dos primeros índices, se encontró una condición de oligotrofia en ambos periodos climáticos, mientras que con el índice compuesto la condición hallada fue de mesotrofia. Así mismo, los resultados del índice trófico planctónico de Barbe (ITP) indicaron un estado de mesotrofia en la época seca y otro de oligotrofia en el periodo de lluvias. El índice de estado trófico ponderado de Carlson (IET) señaló condiciones de mesotrofía en la época seca y de eutrofía en la temporada lluviosa.

La relación N: P se calculó en función de las formas iónicas disueltas en el agua y no con base en las concentraciones totales, en concordancia con lo sugerido por Sierra (2011). Cuando esta relación es mayor a 9, se considera que el fósforo soluble limita el desarrollo de los organismos en el ecosistema acuático (Ramírez \& Viña, 1998). La relación N: $P$ fue menor a 9 en las dos épocas climáticas, lo cual sugiere

Tabla 2. Estado trófico del humedal El Salitre en las dos épocas climáticas según diferentes índices

\begin{tabular}{lrcrc}
\hline Índice & $\begin{array}{c}\text { Época } \\
\text { seca }\end{array}$ & $\begin{array}{c}\text { Estado } \\
\text { trófico }\end{array}$ & $\begin{array}{c}\text { Época } \\
\text { lluviosa }\end{array}$ & $\begin{array}{c}\text { Estado } \\
\text { trófico }\end{array}$ \\
\hline Cianofíceo & 0,24 & Oligotrófico & 0,23 & Oligotrófico \\
\hline Euglenofíceo & 0,32 & Oligotrófico & 0,35 & Oligotrófico \\
Compuesto & 1,48 & Mesotrófico & 1,43 & Mesotrófico \\
ITP & 37,00 & Mesotrófico & 7,00 & Oligotrófico \\
IET ponderado & 53,82 & Mesotrófico & 68,06 & Eutrófico \\
\hline N:P & 2,42 & No aplica & 5,80 & No aplica \\
\hline
\end{tabular}

Los tres primeros corresponden a los índices de Nygaard

ITP= índice trófico planctónico de Barbe, IET= índice ponderado de estado trófico de Carlson, $\mathrm{N}: \mathrm{P}=$ relación nitrógeno: fósforo que en el humedal El Salitre el nitrógeno fue limitante para el crecimiento del fitoplancton. Estos resultados evidencian una deficiencia de nitrógeno, particularmente en la época seca y coinciden con lo reportado para lagos tropicales por Lewis (2002). Los promedios de otras variables físicas y químicas de El Salitre y de otros humedales urbanos de Bogotá se presentan en la tabla 3.

Composición y abundancia del fitoplancton. Durante el estudio se identificaron 114 morfoespecies de algas, pertenecientes a 53 géneros, 33 familias, 24 órdenes y 9 clases. La mayor riqueza se presentó en las clases Conjugatophyceae (=Zignematophyceae, $28 \%$ ) y Chlorophyceae (27\%), seguidas por las clases Bacillariophyceae (15\%) y Euglenophyceae (11 \%). Las cinco clases restantes presentaron pocos taxones (18\%). Las clases con mayor abundancia de individuos fueron Conjugatophyceae (=Zignematophyceae) y Chlorophyceae; las menos abundantes fueron Xanthophyceae y Chrysophyceae (Tabla 4). La clase Conjugatophyceae alcanzó los mayores promedios de abundancia con 2757 ind $\mathrm{mL}^{-1}(43 \%)$ en la temporada seca y 11209 ind $\mathrm{mL}^{-1}$ (78 \%) en la época lluviosa. Para todo el humedal, las especies más representativas fueron la desmidia Heimansia pusilla (5745 inds $\mathrm{mL}^{-1}$ en promedio), la euglenofícea Trachelomonas volvocinopsis (764 ind $\mathrm{mL}-1)$ y la clorofícea Monoraphidium aff. circinale $\left(750 \mathrm{ind} \mathrm{mL}^{-1}\right)$. En el sitio uno, donde la profundidad fue menor y $\mathrm{pH}$ fue un poco más alto, abundaron Lepocinclis spp., Staurastrum spp. y Closterium kuetzingii. En el punto dos, con una mayor transparencia del agua, fueron importantes Scenedesmus spp., Strombomonas spp. y Eunotia spp. Los taxones Stauroneis spp, Cosmarium spp. y Desmodesmus spp. tuvieron mayor representación en el punto tres, donde la saturación de oxígeno fue menor.

Tabla 3. Parámetros físicos y químicos del humedal El Salitre y de otros humedales urbanos de Bogotá muestreados en periodos climáticos similares

\begin{tabular}{|c|c|c|c|c|c|c|c|c|c|c|c|c|c|c|}
\hline \multirow[t]{2}{*}{ Variable } & \multicolumn{2}{|c|}{ EI Salitre } & \multicolumn{2}{|c|}{ Tibanica } & \multicolumn{2}{|c|}{ Jaboque } & \multicolumn{2}{|c|}{$\begin{array}{c}\text { Juan } \\
\text { Amarillo }\end{array}$} & \multicolumn{2}{|c|}{$\begin{array}{c}\text { Santa María } \\
\text { del Lago }\end{array}$} & \multicolumn{2}{|c|}{ Guaymaral } & \multicolumn{2}{|c|}{ Meridor } \\
\hline & $\mathbf{S}$ & $\mathbf{L}$ & $\mathbf{S}$ & $\mathbf{L}$ & $\mathbf{S}$ & $\mathbf{L}$ & $\mathbf{S}$ & $\mathbf{L}$ & $\mathbf{S}$ & $\mathbf{L}$ & $\mathbf{S}$ & $\mathbf{L}$ & $\mathbf{S}$ & $\mathbf{L}$ \\
\hline pH (unidades) & 6,99 & 6,71 & 6,8 & 7,04 & 8,82 & 9,33 & 7,06 & 7,42 & 7,3 & 7,28 & 7,3 & 7,43 & 7,74 & 7,63 \\
\hline Temperatura $\left({ }^{\circ} \mathrm{C}\right)$ & 17,4 & 18,8 & 19,1 & 18,7 & 22,5 & 17,4 & 16,5 & 19,3 & 21,3 & 18,7 & 15,7 & 17,0 & 20,1 & 19,3 \\
\hline Conductividad $\left(\mu \mathrm{S} \mathrm{cm}^{-1}\right)$ & 98,6 & 98,8 & 1840 & 804 & 168 & 173 & 585 & 648 & 294 & 241 & 485 & 395 & 1339 & 1351 \\
\hline Oxígeno disuelto $\left(\mathrm{mg} \mathrm{L}^{-1}\right)$ & 6,2 & 6,6 & 1,05 & 2,04 & 8,57 & 7,94 & 1,3 & 2,43 & 1,94 & 6,88 & 3,19 & 1,23 & 3,5 & 7,21 \\
\hline Alcalinidad $\left(\mathrm{mg} \mathrm{L}^{-1} \mathrm{CaCO}_{3}\right)$ & 55,3 & 48,6 & --- & --- & --- & --- & --- & --- & --- & --- & --- & --- & --- & --- \\
\hline Acidez total $\left(\mathrm{mg} \mathrm{L}^{-1} \mathrm{CaCO}_{3}\right)$ & 10,6 & 16,0 & --- & --- & --- & --- & --- & --- & --- & --- & --- & --- & --- & --- \\
\hline Dureza total $\left(\mathrm{mg} \mathrm{L}^{-1} \mathrm{CaCO}_{3}\right)$ & 56,0 & 83,3 & 179 & 137 & 62 & 54 & 136 & 105 & 120 & 100 & 176 & 84 & 262 & 394 \\
\hline $\mathrm{DBO}_{5}\left(\mathrm{mg} \mathrm{L}^{-1} \mathrm{O}_{2}\right)$ & 9,7 & 11,0 & 8 & 9 & 16 & 19 & 25 & 147 & 10 & 7 & 20 & 198 & 8 & 48 \\
\hline $\mathrm{NO}_{2}\left(\mathrm{mg} \mathrm{L}^{-1}\right)$ & 0,016 & 0,004 & 0 & 0,001 & 0,3 & 0,006 & 7 & 0,008 & 1 & 0,002 & 0 & 0,004 & 0 & 0,103 \\
\hline $\mathrm{NO}_{3}\left(\mathrm{mg} \mathrm{L}^{-1}\right)$ & 0,05 & 0,05 & 0 & 0,03 & 0,3 & 0,18 & 0,3 & 0 & 0 & 0,03 & 0 & 0,04 & 0,2 & 1,33 \\
\hline $\mathrm{NH}_{4}\left(\mathrm{mg} \mathrm{L}^{-1}\right)$ & 0,025 & 0,453 & 0,6 & 1,62 & 0,6 & 1,39 & 14 & 33,84 & 0,7 & 0,48 & 1 & 0,36 & 0 & 3,4 \\
\hline $\mathrm{PO}_{4}\left(\mathrm{mg} \mathrm{L}^{-1}\right)$ & 0,045 & 0,193 & 1,4 & 0,71 & 0,5 & 0,34 & 9,9 & 0,77 & 0 & 0,21 & 0,3 & 1,45 & 0,2 & 0,1 \\
\hline
\end{tabular}

$\mathrm{S}=$ época seca, $\mathrm{L}=$ época lluviosa

Datos de los humedales Tibanica, Jaboque, Juan Amarillo, Santa María del Lago, Guaymaral y Meridor tomados de Castro \& Pinilla (2014) 
Tabla 4. Abundancia promedio relativa (\%) de las clases de algas planctónicas encontradas en el humedal El Salitre en los dos periodos climáticos

\begin{tabular}{lcc}
\hline Clase & Época seca & Época lluviosa \\
\hline Cyanophyceae & 3,7 & 1,8 \\
Chrysophyceae & 0,1 & 0,1 \\
Xanthophyceae & 0,0 & 0,1 \\
Bacillariophyceae & 9,6 & 6,5 \\
Cryptophyceae & 2,6 & 2,1 \\
Dinophyceae & 1,8 & 0,8 \\
Euglenophyceae & 14,1 & 2,2 \\
Chlorophyceae & 24,8 & 7,7 \\
Conjugatophyceae & 43,3 & 78,6 \\
Total & 100,0 & 100,0 \\
\hline
\end{tabular}

\section{Discusión}

Para definir el estado trófico del humedal El Salitre se tuvieron en cuenta cuatro métodos de análisis. El primero consistió en determinar las características fisicoquímicas del agua, particularmente las concentraciones de nutrientes y el segundo se basó en la medición de la concentración de clorofila $a$ fitoplanctónica. En los dos casos, el estado trófico se estableció mediante comparación con escalas definidas en la literatura para cada parámetro. El tercer método se fundamentó en el cálculo de índices basados en distintos parámetros. La cuarta estrategia consistió en relacionar la composición del fitoplancton con el estado trófico del humedal.

La alcalinidad registrada en el humedal El Salitre en ambos periodos climáticos (Tabla 3) indica una baja reserva alcalina y una reducida capacidad buffer del sistema. Esto implica que la adición o extracción de $\mathrm{CO}_{2}$ por procesos respiratorios o fotosintéticos, respectivamente, puede generar variaciones importantes en el pH (Roldán \& Ramírez, 2008). Por otro lado, teniendo en cuenta que el $\mathrm{pH}$ fue cercano a 7,0 se puede deducir que la alcalinidad total estuvo representada fundamentalmente por bicarbonatos (Esteves, 1998; Romero, 2009). La baja acidez registrada en las dos épocas podría indicar una alta actividad fotosintética por parte no solo del fitoplancton, sino también de las macrófitas sumergidas, dado que en el proceso de fotosíntesis el $\mathrm{CO}_{2}$ se utiliza como fuente de carbono (Romero, 2009), lo que reduce la formación de ácido carbónico en el agua. De esta manera, y de acuerdo con el $\mathrm{pH}$ registrado en ambos periodos climáticos, la reducida acidez se debería a la escasa presencia de $\mathrm{CO}_{2}$ disuelto (Romero, 2009).

Según la dureza total, las aguas del humedal El Salitre se clasifican como blandas a moderadamente duras (Romero, 2009), lo cual se relaciona también con la baja alcalinidad medida (Sierra, 2011). De acuerdo con Roldán \& Ramírez (2008), las aguas con baja dureza son poco productivas a nivel biológico. Sin embargo, los resultados de biomasa de clorofila fitoplanctónica muestran que El Salitre tiene una productividad algal importante. Se observó que, al aumentar los valores de dureza en la época de lluvias, también se incrementó la biomasa de clorofila $a$ en este periodo. En relación con otros humedales, la dureza total de El Salitre fue baja, con excepción del humedal Jaboque, donde se han registrado valores similares (Tabla 3 ).

La conductividad eléctrica fue baja en comparación con la reportada para otros humedales de la ciudad (Tabla 3), lo cual indica un nivel de mineralización relativamente bajo y menor presencia de iones disueltos en el agua. Parámetros como el oxígeno disuelto y el porcentaje de saturación de este gas, que es fundamental para el desarrollo de los organismos y para la degradación de la materia orgánica, tuvieron en ambos periodos climáticos valores superiores a los $6 \mathrm{mg} \mathrm{L}^{-1}$ y saturaciones cercanas al $100 \%$. Esta elevada oxigenación del sistema puede deberse en buena parte al proceso de fotosíntesis que realizan tanto las algas fitoplanctónicas como las macrófitas sumergidas (García \& Cruz, 2013). El mantener estos altos niveles de oxígeno en el agua favorece notablemente el desarrollo de las comunidades acuáticas. Como se observa en la tabla 3, las medidas de oxígeno de El Salitre fueron altas en relación con las de otros humedales de Bogotá $\left(<5,5 \mathrm{mg} \mathrm{L}^{-1}\right)$, excepto en Jaboque, donde se registró un promedio de $8,57 \mathrm{mg} \mathrm{L}^{-1}$ en la época seca (Castro \& Pinilla, 2014).

Con respecto a los nutrientes, el incremento del nitrógeno amoniacal en la época de lluvias puede deberse a un aumento en la descomposición de la materia orgánica en condiciones anóxicas (Wetzel, 2001; Sierra, 2011), lo que posiblemente ocurre en el fondo del humedal. Sin embargo, al no tener mediciones de oxígeno disuelto del fondo, esta explicación se plantea a manera de hipótesis. En relación con lo anterior, como se observa en la tabla 3 , en todos los humedales (excepto en Guaymaral) se evidenció el incremento de las concentraciones de nitrógeno amoniacal durante la época de lluvias, lo cual indica que probablemente en este periodo se favorecen los procesos de descomposición de nitrógeno orgánico (Romero, 2009). De acuerdo con el pH (menor a 9), se deduce que en el nitrógeno amoniacal registrado predomina el ión amonio, lo cual es común en aguas naturales (Romero, 2009). Así mismo, teniendo en cuenta que el nitrógeno total también se incrementó en la época de lluvias, se debe considerar que en este periodo el humedal posiblemente recibió aportes de compuestos nitrogenados a través de la escorrentía superficial y del aporte natural de la lluvia (Romero, 2009).

Por otro lado, las bajas concentraciones de nitritos encontradas en las dos épocas climáticas, especialmente en la temporada de lluvias, pueden estar relacionadas con los altos niveles de oxígeno disuelto registrados, lo cual pudo favorecer la oxidación de los nitritos, que de esta manera pasan a nitratos. Se esperaría encontrar mayores niveles de nitratos en el agua, pero las concentraciones medidas en ambos periodos climáticos fueron bajas, lo que posiblemente se explica porque los nitratos se absorben y utilizan 
directamente por parte de las algas y de la vegetación acuática para sintetizar proteínas (Sze, 1993; Roldán \& Ramírez, 2008). Con respecto a otros humedales del Distrito Capital, la concentración de nitratos en El Salitre fue baja en ambos periodos climáticos, e indica que los problemas de eutroficación en este cuerpo de agua no son preocupantes (Sierra, 2011), siempre y cuando se mantengan sus condiciones de aislamiento de las fuentes urbanas de contaminación.

Por su parte, la concentración de ortofosfatos se incrementó durante la época de lluvias, y esto pudo favorecer el aumento de la biomasa fitoplanctónica durante éste periodo, dado que este nutriente se encuentra disuelto en el agua y es aprovechado directamente por las algas (Sierra, 2011; Roldán \& Ramírez, 2008). Se debe considerar además que la asimilación de los fosfatos por parte del fitoplancton se realiza con rapidez (Roldán \& Ramírez, 2008). El incremento de ortofosfatos que se registró en la época de lluvias puede estar relacionado con aportes provenientes de la escorrentía superficial (Romero, 2009) y con la resuspensión del fósforo liberado durante el proceso de descomposición de las macrófitas en el sedimento del ecosistema (Wetzel, 2001). No obstante, la tabla 3 muestra claramente que El Salitre es un humedal con una carga mucho menor de fósforo, en comparación con otros humedales bogotanos. Solo Meridor, ubicado en las afueras de la ciudad, y Santa María del Lago, que ha tenido un proceso de descontaminación de varios años (López, 2012), se asemejan en sus concentraciones bajas de ortofosfatos.

En general, teniendo en cuenta que las concentraciones de nutrientes fueron moderadamente bajas en ambos periodos climáticos (un poco mayores en la época de lluvias), es notable la alta abundancia de fitoplancton encontrada en el humedal, reflejada a su vez en la biomasa de clorofila $a$ fitoplanctónica. Se ha demostrado que las macrófitas sumergidas reducen la abundancia de fitoplancton por la competencia directa por los nutrientes y por la provisión de hábitat para invertebrados fitoplanctófagos (SipaúbaTavares \& Dias, 2014), así como por la reducción en la resuspensión del sedimento (Brothers, Hilt, Attermeyer, Grossart, Kosten, Lischke, et al., 2013), e incluso por efectos alelopáticos (Hilt, 2015). Según Kluijver, Ning, Liu, Jeppesen, Gulati \& Middelburg (2015) el establecimiento de macrófitas sumergidas (y de su perifitón asociado) constituye una manera de controlar el fitoplancton. En general, en los lagos someros dominados por macrófitas el fitoplancton desempeña un papel secundario en la producción de carbono, pero dada su elevada capacidad de respuesta a las oportunidades de crecimiento, en algunas ocasiones puede llegar a ser abundante, en especial si no hay peces planctívoros (Padisák \& Reynolds, 2003). Por lo tanto, es destacable la abundancia de fitoplacton en El Salitre, si se tiene en cuenta la presencia importante de macrófitas sumergidas y emergentes en este ecosistema (García \& Cruz, 2013). Esta situación puede explicarse por una o varias de las siguientes razones:
1. En cuerpos de agua someros se favorece la recirculación eficiente de los nutrientes, debido a que generalmente la columna de agua se mezcla con facilidad y esto remueve los sedimentos del fondo y promueve la resuspensión de compuestos inorgánicos, haciéndolos disponibles para el fitoplancton (Reynolds, 1997; Padisák \& Reynolds, 2003). Así, en sistemas de poca profundidad como El Salitre y los demás humedales de la sabana de Bogotá, este ciclo podría repetirse con frecuencia. Este fenómeno se vería reforzado si estos humedales no cuentan con drenajes externos y están confinado por jarillones, como ocurre con El Salitre. Además, en este ecosistema la infiltración es lenta por la baja permeabilidad del subsuelo (EAAB, 2011).

2. En sistemas palustres como El Salitre y demás humedales de la sabana de Bogotá, existe una estrecha relación entre el fondo (bentos) y la columna de agua. Generalmente en estos ambientes no se presenta estratificación térmica y las macrófitas cubren una proporción importante del área del humedal (López, 2012; García \& Cruz, 2013). Por lo tanto, la acumulación de materia orgánica en el fondo favorece la colonización de más macrófitas, las cuales a su vez ayudan a mantener las características funcionales del ecosistema con respecto al $\mathrm{pH}$, los gases disueltos y los ciclos de nutrientes (Frodge, Thomas \& Pauley, 1991). De esta manera, la actividad respiratoria y de mineralización en el bentos puede liberar las formas inorgánicas de los nutrientes, que pasan a la zona limnética y son aprovechados por las algas planctónicas.

3. Teniendo en cuenta que El Salitre no tiene conexiones de redes de alcantarillado que puedan aportar aguas residuales al sistema (EAAB, 2011), es posible que parte del incremento de los nutrientes (nitrógeno y fósforo) durante la época de lluvias sea generado por la escorrentía de la micro cuenca local, lo cual implica el lavado y transporte de los nutrientes presentes en el suelo circundante hasta el humedal (Romero, 2009); esto favorecería el desarrollo del fitoplancton.

4. Ciertos grupos de algas abundantes en El Salitre, como las desmidiáceas, algunas clorofíceas clorococales y las crisofíceas flageladas, se encuentran generalmente en cuerpos de agua con baja concentración de nutrientes (Bellinger \& Sigee, 2010). Son taxones muy productivos y eficientes con menores cantidades de nitrógeno y fósforo (Reynolds, 2006). La composición biótica fitoplanctónica reflejaría entonces un sistema productivo con una carga no tan elevada de estos elementos.

De acuerdo con lo anterior, la relativamente baja concentración de nutrientes en ambos periodos climáticos indicaría una condición de oligotrofia a mesotrófia en el sistema. Esto parece deberse a que el humedal solamente es alimentado por aguas lluvias y de escorrentía, lo que limita la entrada de nutrientes al cuerpo de agua. Sin embargo, al tener en cuenta los criterios establecidos por Dabrowski (2014) para el ortofosfato y por Esteves (1998) para la clorofila $a$ fitoplanctónica, El Salitre sería 
eutrófico (Tabla 1); la profundidad Secchi $(0,38-0,49 \mathrm{~m})$, por su parte, lo clasificaría como hipereutrófico (OCDE, 1982). Es importante tener en cuenta que los criterios con los cuales se comparan las variables medidas en El Salitre se definieron para zonas templadas y que su aplicación no es clara para cuerpos de agua tropicales (Esteves, 1998). Además, Huszar, Silva, Domingos, Marinho \& Melo (1998) señalan que la concentración de clorofila $a$ fitoplanctónica no es un atributo adecuado para evaluar el estado trófico de los ambientes tropicales poco profundos, bajo los criterios dados para zonas templadas. Es claro que en estos ambientes andinos tropicales someros, como los humedales del altiplano Cundiboyacense, la resuspensión de fósforo mantiene un grado de trofía elevado que podría considerarse como normal. A este respecto aún no se han definido con certeza los límites de las categorías tróficas en la región tropical con base en el fósforo, pero lo datos de El Salitre sugieren que sería necesario incrementar dichos límites. El trabajo de Salas \& Martino (1991) establece una concentración de $0,118 \mathrm{mg} \mathrm{L}^{-1}$ de fósforo total como límite inferior del estado eutrófico, lo cual parece más adecuado para los lagos tropicales. Más recientemente Ortiz-Jiménez, De Anda \& Maniak (2006) señalan que los valores límites para los estados tróficos en lagos cálidos tropicales de América Latina son cuatro veces más altos que los estimados en los lagos templados del hemisferio norte. De esta manera, la clasificación de El Salitre sería de mesotrofia o incluso oligotrofia. Algo similar podría ocurrir con la concentración de clorofila $a$, cuyas concentraciones en los sistemas tropicales podrían ser naturalmente más altas, con lo cual el estado trófico basado en esta variable se debería revisar.

No hay que olvidar que la condición normal de los humedales es la eutrofia y que esta es una característica propia de estos ambientes, bajo la cual funcionan adecuadamente. Precisamente, la biota de los humedales es la encargada de digerir toda la carga orgánica y de nutrientes que tienen este tipo de ecosistemas, razón por la cual los humedales se consideran los riñones de los paisajes en que se encuentran (Mitchell, 1994).

El humedal El Salitre tiene poca profundidad $(<1,5$ m) y como se mencionó, hay una mayor probabilidad de interacción entre el sedimento y la columna de agua. Según Esteves (1998), la disponibilidad de nutrientes en este tipo de ambientes no es un buen indicador del nivel trófico y esto se debe a que las concentraciones de las formas disueltas de nutrientes no reflejan su verdadera disponibilidad, debido a las altas tasas de absorción y ciclado que tienen los cuerpos de aguas tropicales poco profundos. Sumado a esto, el efecto de la disponibilidad de nutrientes sobre el fitoplancton es usualmente complejo de medir, debido a la dificultad de su detección y a las bajas concentraciones de nutrientes que requieren las algas para su crecimiento (Reynolds, 1992).

Los índices basados en las proporciones de taxones de algas se sustentan en la idea de que los atributos del fitoplancton se relacionan con el estado trófico (Huszar, et al., 1998). En El Salitre estos índices indicaron un estado oligotrófico a mesotrófico, coincidente en buena parte con los resultados físicos y químicos. Por su parte, el índice de estado trófico ponderado de Carlson (IET) señaló condiciones de mesotrofía a eutrofía. El IET ponderado contempla para su cálculo parámetros como la profundidad Secchi y la concentración de clorofila $a$ fitoplanctónica, variables que como se discutió, no parecen mostrar adecuadamente el estado trófico en los sistemas tropicales poco profundos. Por esta razón, la composición del fitoplancton puede ser más útil que estos índices tróficos al momento de clasificar el grado de enriquecimiento con nutrientes de los ecosistemas acuáticos tropicales (Huszar, et al., 1998). Teniendo en cuenta estas consideraciones, se puede decir que términos generales, los distintos índices calculados señalan cierta tendencia del humedal El Salitre a la oligotrofia o a la mesotrofia en ambos periodos climáticos.

La buena capacidad del fitoplancton como indicador trófico se debe a que las algas responden a las concentraciones de nutrientes de manera rápida, pues están embebidas directamente en el agua y las condiciones físicas y químicas del medio las afectan directamente (Confederación Hidrográfica del Ebro, 2005). Así, los ensambles que forman ciertos grupos de especies del fitoplancton pueden utilizarse para clasificar el estado trófico de los ecosistemas acuáticos, debido a que las especies reflejan la complejidad de los procesos que se dan en el sistema (Huszar, et al., 1998). Por esta razón, la composición y abundancia de esta comunidad en el humedal El Salitre puede ser un indicador adecuado de su condición trófica.

La dominancia de desmidias en El Salitre es un indicativo evidente de ambientes oligotróficos (Huszar, et al., 1998). Los ensambles fitoplanctónicos de especies de los géneros Staurodesmus, Staurastrum, Cosmarium y Closterium, que fueron representativos en el humedal, son propios de aguas pobres en nutrientes, ligeramente ácidas y de baja alcalinidad (Reynolds, 1998; Huszar, et al., 1998; Bicudo \& Menezes, 2006; Bellinger \& Sigee, 2010; Guiry \& Guiry, 2015). Lo anterior coincide con la clasificación de Hutchinson (1967), ya que en El Salitre fue característico el tipo oligotrófico desmidiáceo. De igual manera, Heimansia pusilla, la especie más abundante durante el estudio, se ha registrado en sistemas mesotróficos de aguas ácidas (Coesel, 1993; Pinilla, 2000). Esta desmidia es de hábitos ticoplanctónicos (asociada a raíces de plantas acuáticas) y con preferencia por aguas no muy mineralizadas (Negro, De Hoyos \& Aldasoro, 2003), como es el caso del humedal El Salitre.

De las clorofíceas, las clorococales tuvieron una gran representatividad a nivel de riqueza, y dos especies del género Monoraphidium fueron las más abundantes de este grupo. Aunque éste es uno de los géneros más cosmopolita entre las clorococales, predomina principalmente en ambientes oligo a mesotróficos (Bicudo \& Menezes, 2006). Dentro de las euglenofitas, el género Trachelomonas, que está asociado con aguas mesotróficas poco profundas 
(Reynolds, 2006), tuvo la mayor abundancia dentro de esta clase, en especial $T$. volvocinopsis en la época seca. A pesar de que los géneros Dinobryon (Chrysophyceae) y Mallomonas (Xanthophyceae) tuvieron baja abundancia, se debe tener en cuenta que generalmente se encuentran en ambientes oligotróficos o mesotróficos de aguas blandas (Reynolds, 1997; Reynolds, 2006).

El humedal Santa María del Lago, que según López (2012) es mesotrófico a eutrófico, Rodríguez (2012) encontró que las euglenofitas fueron la clase más abundante (>80\%), con las especies Trachelomonas volvocina, Phacus longicauda, P. orbicularis, P. helikoides y Lepocinclis ovum como las más representativas, seguidas por las clorofíceas Crucigenia quadrata, Dictyosphaerium pulchellum y Oocystis sp. Este ensamble tiene cierta similitud con la composición de El Salitre, pero es evidente que en Santa María la dominancia de euglenofitas señala un trofismo de mayor grado. En el humedal Córdoba se han hallado condiciones de eutrofia a hipereutrofía (SDA \& UMNG, 2008), con prevalencia de taxones perifíticos de las clases Bacillariophyceae, Cyanophyceae y Euglenophyceae. Los géneros más representativos en Córdoba fueron Synechococcus, Merismopedia y Nitzschia. En el humedal Jaboque, Arcos \& Gómez (2006) utilizaron sustratos artificiales para evaluar la colonización de algas perifíticas y encontraron que las clases más representativas fueron Euglenophyceae, Cyanophyceae y Bacillariophyceae, resultados que asociaron con la condición de eutrofía de ese cuerpo de agua. En el humedal Juan Amarillo, considerado como eutrófico (CIC \& EAAB, 2008), Rodríguez (2012) encontró que en la época seca dominaron las clases Dinophyceae y Euglenophyceae, mientras que en la época lluviosa fueron más representativas las Chlorophyceae $(>50 \%)$, Euglenophyceae, Dinophyceae y Bacillariophyceae. Entre las especies más importantes en Juan Amarillo, Rodríguez (2012) registró a Ceratium furcoides, Peridinium cinctum, Trachelomonas hispida, T. volvocina, Lepocinclis salina, P. longicauda, Monoraphidium sp, Closteriopsis sp. y Oocystis sp. Como se ve, en Córdoba, Jaboque y Juan Amarillo, considerados como humedales urbanos de Bogotá eutróficos o hipereutróficos, la composición fitoplanctónica fue muy diferente a la de El Salitre, un sistema con un grado de trofía menor.

Los estudios disponibles muestran que la composición algal de la mayoría de los humedales de Bogotá, con predominio de las clases Euglenophyceae y Cyanophyceae, está asociada a condiciones de eutrofia. Generalmente estos grupos prosperan en ambientes acuáticos con altas concentraciones de nutrientes y ricos en materia orgánica (Ramírez, 2000). Son notorias las diferencias en la composición y dominancia de las especies del fitoplancton de El Salitre con respecto a las de otros humedales del Distrito Capital. En El Salitre las clases con mayor riqueza fueron Conjugatophyceae, particularmente las desmidias, y Chlorophyceae, con predominio de las clorococales. La ausencia de aportes urbanos de nutrientes y el ingreso de aguas lluvias y de escorrentía (EAAB, 2011) explicarían esta situación ecológicamente más saludable del humedal El Salitre. Los demás humedales de la ciudad reciben en mayor o menor grado cargas de contaminación por aguas residuales, tanto de origen doméstico como industrial (SDA, 2008), lo cual acelera los procesos de eutrofización en estos ecosistemas, que se reflejan en la composición de sus comunidades de algas planctónicas.

\section{Conclusiones}

Las concentraciones de nutrientes del humedal El Salitre fueron bajas en relación con otros humedales urbanos de Bogotá D.C. e indicaron una condición de oligotrofia a mesotrofía para los compuestos nitrogenados y de supuesta eutrofía para los ortofosfatos, siendo el nitrógeno el nutriente limitante. Las clases dominantes del fitoplancton fueron Conjugatophyceae (desmidiáceas) y Chlorophyceae (clorococales), con especies que indicaron condiciones de oligotrófia a mesotrófia en el sistema. La hipótesis propuesta inicialmente se pudo comprobar en cuanto al estado trófico menor del humedal El Salitre, en comparación con los demás humedales de Bogotá. Los grupos fitoplanctónicos dominantes en este humedal corresponden a taxones algales específicos de aguas con poca a moderada cantidad de nutrientes. Esto hace de El Salitre un sistema que podría servir de referencia para los demás humedales de la ciudad $\mathrm{y}$ que por lo tanto requiere un manejo que preserve sus condiciones actuales. Sus características físicas, químicas y fitoplanctónicas podrían servir de meta a alcanzar en la planificación de proyectos de rehabilitación ambiental de otros humedales y para la posterior evaluación de dichos proyectos, como lo sugieren los principios de restauración ecológicos de SER (2004).

\section{Agradecimientos}

El proyecto fue financiado en parte por el Programa de Estímulos a la Investigación "Thomas van der Hammen" del Jardín Botánico de Bogotá José Celestino Mutis. Además, los autores agradecen al grupo de investigación "Biodiversidad, biotecnología y conservación de ecosistemas" del Departamento de Biología de la Universidad Nacional de Colombia por el apoyo logístico y al Laboratorio de Limnología de la Universidad Jorge Tadeo Lozano donde se hicieron los análisis químicos y biológicos. Se agradece también a la Secretaría Distrital de Ambiente de la Alcaldía Mayor de Bogotá por los permisos otorgados para la toma de muestras.

\section{Conflicto de intereses}

Los autores declaran no tener conflicto de intereses

\section{Referencias}

Alcaldía Local de Barrios Unidos. 2014. Plan Ambiental Local 2013-2016. Bogotá: Alcaldía Local de Barrios Unidos. 
Alcaldía Mayor de Bogotá. 2000. Historia de los humedales de Bogotá. Disponible en: www.bogota.gov.co/guia/interfaz/ usuario/anexos/Humedales.doc, accedido en septiembre de 2016

American Public Health Association (APHA), American Water Works Association (AWWA), Water Pollution Control Federation (WPCF). 2012. Standard methods for the examination of water and wastewater (22nd ed.). Washington: USA: American Public Health Association.

Arcos, M.P., Gómez, A.C. 2006. Microalgas perifíticas como indicadoras del estado de las aguas de un humedal urbano: Jaboque, Bogotá D.C., Colombia. Nova. 4 (6): 60-79.

Bellinger, E., Sigee, D. 2010. Freshwater algae: identification and use as bioindicators. Chichester, UK: Wiley-Blackwell.

Beltrán, B.J., Rangel, J.E. 2013. Modelación del estado trófico del humedal de Jaboque, Bogotá D.C., Colombia. Acta Biológica Colombiana. 18 (1): 149-164.

Bicudo, C., Meneses, M. 2006. Gêneros de algas de águas continentais do Brasil: chave para identificação e descrições (2da ed.). São Carlos, Brasil: Rima.

Brothers, S.M., Hilt,S., Attermeyer,K., Grossart, H.P., Kosten, S., Lischke, B., Mehner,T., Meyer,N., Scharnweber, K., Köhler, J. 2013. A regime shift from macrophyte to phytoplankton dominance enhances carbon burial in a shallow, eutrophic lake. Ecosphere. 4 (11): 1-17.

Castro, D., Pinilla, G.A. 2014. Periphytic diatom index for assessing the ecological quality of the Colombian Andean urban wetlands of Bogotá. Limnética. 33 (2): 297-312.

Conservación Internacional Colombia (CIC), Empresa de Acueducto y Alcantarillado de Bogotá (EAAB). 2008. Convenio "Investigación aplicada en restauración ecológica”. Humedal Juan Amarillo. Informe final. Bogotá, Colombia: Conservación Internacional Colombia y Empresa de Acueducto y Alcantarillado de Bogotá.

Coesel, P.F. 1993. Taxonomic notes on Dutch desmids II. Cryptogamie, Algologie. 14: 105-114.

Confederación Hidrográfica del Ebro. 2005. Metodología para el establecimiento del estado ecológico según la Directiva Marco del Agua. Protocolos de muestreo y análisis para fitoplancton. Madrid: Ministerio de Medio Ambiente de España.

Dabrowski, J.M. 2014. Applying SWAT to predict orthophosphate loads and trophic status in four reservoirs in the upper Olifants catchment, South Africa. Hydrology and Earth Systems Science. 18: 2629-2643.

Departamento Técnico Administrativo del Medio Ambiente (DAMA). 2002. Los humedales del altiplano en Bogotá, bases técnicas para su conservación, restauración y manejo. Documento Técnico. Bogotá: Departamento Administrativo del Medio Ambiente, Alcaldía Mayor de Bogotá.

De la Fuente, M.J. (ed). 2004. Metodología para el establecimiento del estado ecológico según la Directiva Marco del Agua en la Confederación Hidrográfica del Ebro. Zaragoza: Ministerio de Medio Ambiente de España. Disponible en: http:// www.mapama.gob.es/es/agua/publicaciones/Protocolos muestreo_biologico_con_portada_tcm7-16059.pdf

Empresa de Acueducto y Alcantarillado de Bogotá (EAAB). 2011. Balance hidroclimático del cuerpo de agua ubicado en el Parque El Salitre de la ciudad de Bogotá. Bogotá: Empresa de Acueducto y Alcantarillado de Bogotá.
Esteves, F.A. 1998. Fundamentos de limnologia (2da ed.). Rio de Janeiro: Interciência/FINEP.

Frodge, J.D., Thomas, G.L., Pauley, G.B. 1991. Sediment phosphorus loading beneath dense canopies of aquatic macrophytes. Lake and Reservoir Management. 7 (1): 61-71.

García, B.D., Cruz, A.S. 2013. Propuesta de un plan de manejo ambiental del humedal El Salitre, Bogotá D.C. (Tesis de especialistas). Universidad Distrital Francisco José de Caldas. Bogotá.

Guiry, M.D., Guiry, G.M. 2015. AlgaeBase. World-wide electronic publication, National University of Ireland, Galway. Disponible en http://www.algaebase.org., accedida entre enero y junio de 2015.

Hilt, S. 2015. Regime shifts between macrophytes and phytoplankton - concepts beyond shallow lakes, unravelling stabilizing mechanisms and practical consequences. Limnetica. 34 (2): 467-480.

Huszar, V., Silva, L.H., Domingos, P., Marinho, M., Melo, S. 1998. Phytoplankton species composition is more sensitive than OECD criteria to the trophic status of three Brazilian tropical lakes. Hydrobiologia. 369/370: 59-71.

Hutchinson, G.E. 1967. A treatise on limnology, Vol II. Introduction to lake biology and the limnoplankton. New York: John Wiley \& Sons.

Karlson, B., Cusack, C., Bresnan, E. (eds). 2010. Microscopic and molecular methods for quantitative phytoplankton analysis. Paris: UNESCO (IOC Manuals and Guides, $N^{\circ}$. 55.) $(\mathrm{IOC} / 2010 / \mathrm{MG} / 55)$

Kluijver, A. Ning, J., Liu, Z., Jeppesen, E., Gulati, R.D., Middelburg, J.J. 2015. Macrophytes and periphyton carbon subsidies to bacterioplankton and zooplankton in a shallow eutrophic lake in tropical China. Limnology and Oceanography. 60: 375-385.

Lewis, W.M. 2002. Causes for the high frequency of nitrogen limitation in tropical lakes. Verhandlungen der Internationalen Vereinigung der Limnologie. 28: 210-213.

López, P.R. 2012. Estado trófico de un humedal urbano andino tropical: Santa María del Lago Bogotá D.C., Colombia. Bogotá: Universidad Militar Nueva Granada.

Malagón, A. 2008. Descripción del estado trófico de cuatro humedales de la Sabana de Bogotá, a partir de la composición, biomasa y producción del fitoplancton (Tesis pregrado). Universidad Nacional de Colombia. Bogotá.

Moreno, V., García, J.F., Villalba, J.C. 2005. Descripción general de los humedales de Bogotá. Bogotá: Sociedad Geográfica de Colombia. Disponible en: http://www.sogeocol.edu.co/ documentos/humed.pdf, accedido en septiembre de 2016.

Mitchell, D.S. 1994. Floodplain wetlands of the Murray-Darling Basin: management, issues and challenges. In: T. Sharley, C. Huggin (eds.). Murray-Darling Basin floodplain wetlands management. (pp. 1-5). Albury, Australia: Proceedings of the Floodplain Wetlands Management Workshop.

Negro, A.I., De Hoyos, C., Aldasoro, J.J. 2003. Diatom and desmid relationships with the environment in mountain lakes and mires of NW Spain. Hydrobiologia. 505: 1-13.

Organización para la Cooperación y el Desarrollo Económicos (OCDE). 1982. Eutrophication of waters. Monitoring assessment and control. Paris: Organisation de Coopération et Développement Économiques. 
Ortiz-Jiménez, M.A., De Anda, J., Maniak, U. 2006. Estimation of trophic states in warm tropical lakes of reservoirs of Latin America by using GPSS simulation. Interciencia. 31 (5): 345-350.

Padisák, J., Reynolds, C.S. 2003. Shallow lakes: the absolute, the relative, the functional and the pragmatic. Hydrobiologia. 506-509: 1-11.

Parra, O., González, M., Dellarossa, V., Rivera, P., Orellana, M. 1982. Manual taxonómico del fitoplancton de aguas continentales con especial referencia al fitoplancton de Chile. I: Cyanophyceae, II: Chrysophyceae, III: Cryptophyceae, IV: Bacillarophyceae, V: Chlorophyceae. Concepción: Universidad de Concepción.

Pinilla, G. 2000. Indicadores biológicos en ecosistemas acuáticos continentales de Colombia. Compilación bibliográfica. Bogotá: Universidad Jorge Tadeo Lozano.

Pinilla, G. 2010. An index of limnological conditions for urban wetlands of Bogotá city, Colombia. Ecological Indicators. 10 (4): 848-856.

Prescott, G.W., Croasdale, H., Vinyard, W.C. 1972. North American Flora: Desmidiales. Part I. Saccodermae, Mesotaeniaceae. New York: New York Botanical Garden.

Prescott, G.W., Croasdale, H., Vinyard, W.C. 1975. A synopsis of North American Desmids. Part II. Desmidiaceae: Placodermae. Section 1. Lincoln, USA: University of Nebraska Press.

Prescott, G.W., Croasdale, H., \& Vinyard, W.C. (1977). A synopsis of North American Desmids. Part II. Desmidiaceae: Placodermae. Section 2. Lincoln: University of Nebraska Press.

Prescott, G.W., Croasdale, H., Vinyard, W.C., Bicudo, C.E.M. 1981. A synopsis of North American Desmids. Part II. Desmidiaceae: Placodermae. Section 3. Lincoln: University of Nebraska Press.

Prescott, G.W., Bicudo, C.E.M., Vinyard, W.C. 1982. A synopsis of North American Desmids. Part II. Desmidiaceae: Placodermae. Section 4. Lincoln: University of Nebraska Press.

Ramírez, A., Viña, G. 1998. Limnología colombiana. Aportes a su conocimiento y estadísticas de análisis. Bogotá: BP Exploration Company (Colombia), Fundación Universidad de Bogotá Jorge Tadeo Lozano.

Ramírez, J. 2000. Fitoplancton de agua dulce: aspectos ecológicos, taxonómicos y sanitarios. Medellín: Editorial Universidad de Antioquia.

Reynolds, C.S. 1992. Eutrophication and the management of planktonic algae: what Vollenweider couldn't tell us. In: D.W. Sutcliffe, J.G. Jones (eds.). Eutrophication: research and application to water supply. (pp. 4-29). Ambleside, UK: Freshwater Biological Association.

Reynolds, C.S. 1997. Vegetation process in the pelagic: a model for ecosystem theory. Oldendorf/Luhe, Germany: Ecology Institute.

Reynolds, C.S. 1998. What factors influence the species composition of phytoplankton in lakes of different trophic status? Hydrobiologia. 369/370: 11-26.
Reynolds, C.S. 2006. Ecology of phytoplankton. Cambridge: Cambridge University Press.

Rivera-Usme, J., Pinilla-Aguelo, G.A., Rangel-Ch, J.O. 2013. Ensamblaje de macroinvertebrados acuáticos y su relación con las variables físicas y químicas en el humedal de Jaboque-Colombia. Caldasia. 35 (2): 389-408.

Roldán, G., Ramírez, J. 2008. Fundamentos de limnología neotropical (2da ed). Medellín: Editorial Universidad de Antioquia.

Romero, J.A. 2009. Calidad del agua (3ra ed.). Bogotá: Escuela Colombiana de Ingeniería.

Rodríguez, L. 2012. Determinación del estado trófico de tres ecosistemas lénticos de la Sabana de Bogotá con base al fitoplancton, en dos periodos climáticos contrastantes (Tesis pregrado). Universidad Militar Nueva Granada. Bogotá.

Salas, H.J., Martino, P. 1991. A simplified phosphorus trophic state model for warm-water tropical lakes. Water Research. 25 (3): 341-350.

Society for Ecological Restoration International (SER). 2004. Principios de SER International sobre la restauración ecológica. Tucson: Society for Ecological Restoration International. Disponible en http://www.ser.org/docs/defaultdocument-library/spanish.pdf, accedida en junio de 2016.

Secretaría Distrital de Ambiente (SDA). 2008. Protocolo de recuperación y rehabilitación ecológica de humedales en centros urbanos. Bogotá: Secretaria Distrital de Ambiente, Alcaldía Mayor de Bogotá.

Secretaría Distrital de Ambiente (SDA), Universidad Militar Nueva Granada (UMNG). 2008. Formulación del plan de ordenación y manejo de la cuenca del río Salitre en el perímetro urbano del Distrito Capital. Informe de la fase de diagnóstico. Convenio interadministrativo No. 080 del 28 de diciembre de 2007. Bogotá: Secretaría Distrital de Ambiente, Universidad Militar Nueva Granada.

Sierra, C.A. 2011. Calidad del agua, evaluación y diagnóstico. Medellín: Editorial Universidad de Medellín.

Sipaúba-Tavares, LH., Dias, S.G. 2014. Water quality and communities associated with macrophytes in a shallow water-supply reservoir on an aquaculture farm. Brazilian Journal of Biology. 74 (2): 420-428.

Sze, P. 1993. A biology of the algae (2nd ed.). Dubuque: Wm. C. Brown Publishers.

Tell, G., Conforti, V. 1986. Euglenophyta pigmentadas de la Argentina. Berlin-Stuttgart: J.Cramer.

Vásquez, C., Ariza, A., Pinilla, G. 2006. Descripción del estado trófico de diez humedales del altiplano Cundiboyacense. Universitas Scientiarum. 11 (2): 61-75.

Wehr, J.D., Sheath, R.G., Kociolek, R.P. (eds.). 2003. Freshwater Algae of North America. San Diego: Academic Press.

Wetzel, R.G. 2001. Limnology: lake and river ecosystems (3th ed.). Philadelphia: San Diego: Academic Press.

Wetzel, R.G., Likens, G E. 2000. Limnological analyses (3rd ed.). New York: Springer-Verlag. 